\title{
Changing molecular epidemiology of methicillin-resistant Staphylococcus aureus in an Algerian hospital
}

\author{
Patrick Basset ${ }^{1}$, Wahiba Amhis ${ }^{2}$, Dominique S Blanc ${ }^{1,3}$ \\ ${ }^{1}$ Service of Hospital Preventive Medicine, Lausanne University Hospital, Switzerland \\ ${ }^{2}$ Central Laboratory of Biology, Bologhine Ibn Ziri Hospital, Algiers, Algeria \\ ${ }^{3}$ Institute of Microbiology, Lausanne University Hospital, Switzerland
}

\begin{abstract}
Introduction: Methicillin-resistant Staphylococcus aureus (MRSA) is a major cause of both hospital- and community-acquired infections worldwide. However, data about the molecular epidemiology of MRSA in North Africa are still scarce.

Methodology: All MRSA isolates recovered between January 2006 and July 2011 from one Algerian hospital were genetically and phenotypically characterized.

Results: The predominance of a European community-associated-MRSA (CA-MRSA) clone (ST80-SCCmec IV-PVL positive) was revealed by this analysis.

Conclusion: Our data suggest that a CA-MRSA clone recently invaded the hospital setting in Algiers and replaced a typical hospitalassociated pandemic clone such as the Brazilian clone (ST239-SCCmec IIImercury-PVL negative).
\end{abstract}

Key words: MRSA; molecular epidemiology; Algeria; DLST.

J Infect Dev Ctries 2015; 9(2):206-209. doi:10.3855/jidc.4620

(Received 24 December 2013 - Accepted 09 March 2014)

Copyright (C) 2015 Basset et al. This is an open-access article distributed under the Creative Commons Attribution License, which permits unrestricted use, distribution, and reproduction in any medium, provided the original work is properly cited.

\section{Introduction}

Methicillin-resistant Staphylococcus aureus (MRSA) is a major cause of both hospital and community-acquired infections worldwide. However, there is a considerable epidemiological variation of MRSA at both regional and global levels [1]. For example, Mediterranean countries likely represent a hyperendemic region for MRSA whereas the proportion of MRSA in northern Europe is much lower [2]. In particular, the proportion of MRSA in southern Mediterranean countries (e.g., Egypt, Tunisia and Algeria) showed a recent spectacular increase [2]. Nevertheless, data about the molecular epidemiology of MRSA in North Africa are still scarce compared to European countries.

\section{Methodology}

In this study, all MRSA isolates (one per patient; $\mathrm{n}$ $=84$ ) recovered between January 2006 and July 2011 from the Bologhine Ibn Ziri University hospital (250 beds) located in Algiers (Algeria) were characterized. The epidemiological characteristics of these isolates are indicated in Table 1-S. Each bacterial isolate was identified with standard bacteriological procedures (i.e., Gram stain, colonial/morphological appearance, tube coagulase, catalase and DNase tests); susceptibility to 12 common antimicrobial agents (Table 1) was tested using the disc-diffusion method according to the Clinical and Laboratory Standards Institute (CLSI) guidelines [3].

The genetic diversity of this MRSA population was assessed with the highly discriminatory Double Locus Sequence Typing (DLST) method as previously described $[4,5]$. This method is based on the analysis of partial sequences (about 500 base pairs) of the variable $c l f B$ and spa genes. Additionally, the staphylococcal cassette chromosome (SCCmec) element was determined in each strain using a multiplex PCR method described by Kondo et al.; the presence of Panton-Valentine-leukocidin (PVL) genes was tested as previously described $[6,7]$.

\section{Results and discussion}

As expected, all MRSA isolates were resistant to penicillin, oxacillin and cefoxitin (Table 1). In contrast, all isolates were susceptible to rifampin, vancomycin and teicoplanin. Variable resistance rates were observed for kanamycin $(77.4 \%)$, tetracycline 
(58.3\%), clindamycin $(9.5 \%)$, co-trimoxazole $(23.8 \%)$, ofloxacin (15.5\%) and fusidic acid (53.6\%).

A total of 13 clfB and 5 spa alleles were observed among the 84 MRSA isolates typed by DLST and these accounted for 13 different DLST types. eBURST software was used to cluster DLST types sharing at least one identical allele (http://eburst.mlst.net/). There is generally a good concordance between DLST clusters and MLST clonal complexes and it is possible to relate these clusters with international clones [8]. This analysis identified two main clusters as well as three singletons (Figure 1). First, the cluster with DLST ancestor $415-26$ represents $85.7 \%(72 / 84)$ of all isolates. This cluster corresponds to the European CAMRSA clone (ST80) and as expected all isolates of this clone were PVL positive [5]. In addition, most isolates of this clone carry the SCCmec type IV although it was not possible to identify the ccr type in two isolates. Second, the clone with DLST type 28-30 represents $8.3 \%(7 / 84)$ of all isolates. This cluster is related to the Brazilian clone (ST239) and as expected all the isolates of this clone carried the SCCmec IIImercury and were PVL negative [5]. Finally, the remaining five isolates $(6.0 \%)$ belonged to three single DLST types and could not be associated with international clones. All these isolates carried the SCCmec IV and were PVL negative.

The European CA-MRSA (ST80-IV-pos) is the most common community acquired clone in Europe $[9,10]$. Although this clone has been recovered from many European countries, it has often been associated with patients with Middle Eastern or North African origin $[9,10]$. This is probably explained by the predominance of the European CA-MRSA clone in Algeria and in surrounding North African countries
Figure 1. DLST single-locus variant clustering of 84 S. aureus isolates from the Bologhine Ibn Ziri University hospital in Algeria using eBURST. Each circle represents one DLST type, and the diameter of the circle reflects the frequency (i.e., the number of isolates) of that type. Linked DLST types differ at one of the two loci ( $c l f B$ or $s p a)$. SCCmec type is indicated next to each DLST type ("Un" indicates isolates with unknown ccr type) and each DLST types including PVL-positive isolates are indicated by asterisks. Two isolates had a null allele (i.e. 0) at the spa locus. The name of the international clone associated with each of the main cluster is indicated under the cluster.

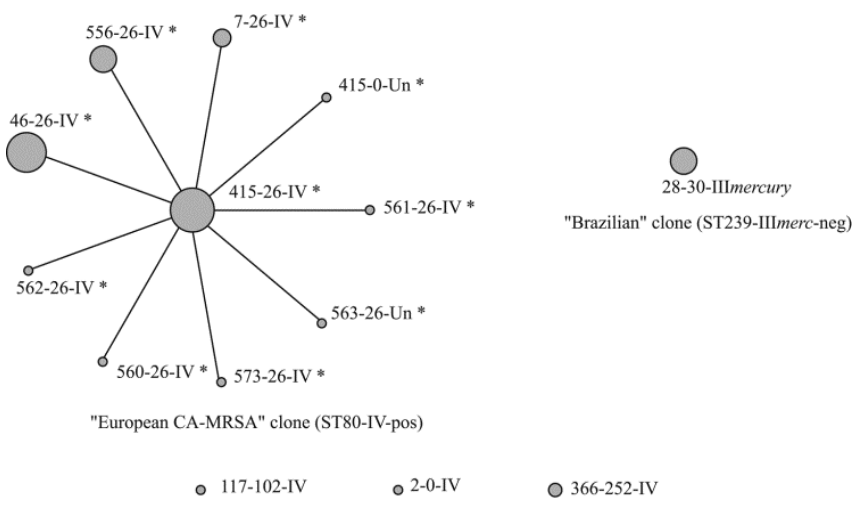

[11-15]. Even though this clone is generally associated with community-acquisitions, our data did not allow discrimination between hospital and community acquisitions. Nevertheless, the widespread occurrence of this clone in Algerian hospitals indicates it has invaded the hospital setting. Moreover, the proportion of isolates belonging to ST80 changed during our study period. Whereas a similar proportion of the Brazilian and European CA-MRSA clone was observed in 2006 (36\% vs. 43\%), the European CAMRSA clone accounted for $>90 \%$ of the isolates in subsequent years (Table 2). This observation as well as similar previous observations suggest a recent

Table 1. Patterns of susceptibility of the European CA-MRSA and Brazilian clones as well as overall Algerian MRSA isolates.

\begin{tabular}{cccc}
\hline & & Resistant isolates (\%) among: & \\
\cline { 2 - 4 } & $\begin{array}{c}\text { European CA-MRSA isolates } \\
\text { (ST80) } \\
(\mathbf{n = 7 2 )}\end{array}$ & $\begin{array}{c}\text { Brazilian MRSA isolates } \\
(\text { ST239) } \\
(\mathbf{n = 7 )}\end{array}$ & $\begin{array}{c}\text { All isolates } \\
(\mathbf{n}=\mathbf{8 4})\end{array}$ \\
Antibiotic & 100 & 100 & 100 \\
Penicillin & 100 & 100 & 100 \\
Oxacillin & 100 & 100 & 100 \\
Cefoxitin & 69.3 & 100 & 77.4 \\
Kanamycine & 55.6 & 71.4 & 58.3 \\
Tetracycline & 8.3 & 0 & 9.5 \\
Clindamycin & 20.8 & 57.1 & 23.8 \\
Co-trimoxazole & 8.3 & 71.4 & 15.5 \\
Ofloxacine & 0 & 0 & 0 \\
Rifampin & 0 & 0 & 0 \\
Vancomycin & 0 & 0 & 0 \\
Teichoplanine & 55.6 & 42.9 & 53.6 \\
Fusidic acid & & & 0 \\
\hline
\end{tabular}


Table 2. Number of isolates belonging to the European CA-MRSA and Brazilian clone per year.

\begin{tabular}{ccccc}
\hline Year & $\begin{array}{c}\text { European CA-MRSA } \\
\text { clone }\end{array}$ & Brazilian clone & Other & Overall \\
\hline 2006 & 6 & 5 & 3 & 0 \\
2007 & 9 & 0 & 1 & 9 \\
2008 & 19 & 0 & 0 & 20 \\
2009 & 26 & 0 & 0 & 8 \\
2010 & 6 & 2 & 1 & 7 \\
2011 & 6 & 0 & $\mathbf{5}$ & $\mathbf{8 4}$ \\
Overall & $\mathbf{7 2}$ & $\mathbf{7}$ & & \\
\hline
\end{tabular}

emergence of the ST80 clone in Algerian hospitals and indicate that this community associated clone is able to quickly replace typical hospital-associated clones such as the Brazilian clone $[12,14]$.

To compare the antibiotic susceptibility and virulence patterns of the major clones recovered in this study with international clones, we analyzed two representative Algerian isolates of the European CAMRSA clone and one Algerian isolate of the Brazilian clone with the Alere StaphyType DNA microarray (Alere Technologies, Jena, Germany) [16]. This microarray targets approximately 170 distinct genes and it was used following protocols and procedure previously described into detail [16]. Interestingly, the presence/absence of resistance and virulence genes for each major Algerian clone was similar to other isolates of the same clone as reported by Monecke (Table 2-S) [16]. This confirms the general homogeneity among Algerian and European isolates of the ST80 clone [10].

In conclusion, our data confirmed the predominance of the PVL-positive European CAMRSA (ST80-IV-pos) clone in Algeria. In addition, our findings suggest that this clone recently replaced other hospital associated pandemic clones in Algerian hospitals.

\section{Acknowledgements}

We are grateful to Caroline Choulat for technical assistance in Switzerland and to Samira Benmesbah for her help in the collection of Algerian S. aureus isolates.

\section{References}

1. Grundmann H, Ires-de-Sousa M, Boyce J, Tiemersma E (2006) Emergence and resurgence of meticillin-resistant Staphylococcus aureus as a public-health threat. The Lancet 368: 874-885.

2. Borg M, de Kraker M, Scicluna E, van de Sande-Bruinsma N, Tiemersma E, Monen, J, Grundmann H; ARMed Project Members and Collaborators (2007) Prevalence of methicillinresistant Staphylococcus aureus (MRSA) in invasive isolates from southern and eastern Mediterranean countries. J Antimicrob Chemother 60: 1310-1315.

3. Clinical and Laboratory Standards Institute (2010) Performance standards for antimicrobial susceptibility testing. Twentieth informational supplement. Clinical and Laboratory Standards Institute, Wayne, PA.

4. Kuhn G, P. Francioli, Blanc DS (2007) Double-locus sequence typing using $c l f B$ and $s p a$, a fast and simple method for epidemiological typing of methicillin-resistant Staphylococcus aureus. Journal of Clinical Microbiology 45: 54-62.

5. Basset P, Senn L, Prod'hom G, Bille J, Francioli P, Zanetti G, Blanc DS (2010) Usefulness of double locus sequence typing (DLST) for regional and international epidemiological surveillance of methicilin-resistant Staphylococcus aureus. Clin Microbiol Infect 16: 1289-1296.

6. Kondo Y, Ito T, Ma XX, Watanabe S, Kreiswirth BN, Etienne J, Hiramatsu K (2007) Combination of Multiplex PCRs for Staphylococcal Cassette Chromosome mec Type Assignment: Rapid Identification System for mec, ccr, and Major Differences in Junkyard Regions. Antimicrobial Agents and Chemotherapy 51: 264-274.

7. Lina G, Piemont Y, Godail-Gamot F, Bes M, Peter MO, Gauduchon V, Vandenesch F, Etienne J (1999) Involvement of Panton-Valentine leukocidin-producing Staphylococcus aureus in primary skin infections and pneumonia. Clin Infect Dis 29: 1128-1132.

8. Basset P, Hammer NB, Kuhn G, Vogel V, Sakwinska O, Blanc DS (2009) Staphylococcus aureus clfB and spa alleles of the repeat regions are segregated into major phylogenetic lineages. Infect Genet Evol 9: 941-947.

9. Tristan A, Bes M, Meugnier H, Lina G, Bozdogan B, Courvalin P, Reverdy ME, Enright MC, Vandenesch F, Etienne J (2007) Global distribution of Panton-Valentine leukocidin-positive methicillin-resistant Staphylococcus aureus, 2006. Emerging Infectious Diseases 13: 594-600.

10. Goering RV, Larsen AR, Skov R, Tenover FC, Anderson KL Dunman PM (2009) Comparative genomic analysis of European and Middle Eastern community-associated methicillin-resistant Staphylococcus aureus (CC80:ST80-IV) 
isolates by high-density microarray. Clin Microbiol Infect 15: 748-755.

11. Ramdani-Bouguessa N, Bes M, Meugnier H, Forey F, Reverdy ME, Lina G, Vandenesch F, Tazir M, Etienne J (2006) Detection of methicillin-resistant Staphylococcus aureus strains resistant to multiple antibiotics and carrying the Panton-Valentine leukocidin genes in an Algiers hospital. Antimicrob Agents Chemother 50: 1083-1085.

12. Bekkhoucha SN, Cady A, Gautier P, Itim F, Donnio PY (2009) A portrait of Staphylococcus aureus from the other side of the Mediterranean Sea: molecular characteristics of isolates from Western Algeria. Eur J Clin Microbiol Infect Dis 28: 553-555.

13. Antri K, Rouzic N, Boubekri I, Dauwalder O, Beloufa A, Ziane H, Djennane F, Neggazi M, Benhabyles B, Bes M, Tazir M, Etienne J, Ramdani-Bouguessa N (2010) High prevalence of community- and hospital-acquired infections of methicillin-resistant Staphylococcus aureus containing Panton-Valentine leukocidin gene in Algiers. Pathol Biol 58: e15-20.

14. Antri K, Rouzic N, Dauwalder O, Boubekri I, Bes M, Lina G, Vandenesch F, Tazir M, Ramdani-Bouguessa N, Etienne J (2011) High prevalence of methicillin-resistant Staphylococcus aureus clone ST80-IV in hospital and community settings in Algiers. Clin microbiol Infect 17: 526532.
15. Ben Slama K, Gharsa H, Klibi N, Jouini A, Lozano C, Gomez-Sanz E, Zarazaga M, Boudabous A, Torres C (2011) Nasal carriage of Staphylococcus aureus in healthy humans with different levels of contact with animals in Tunisia: genetic lineages, methicillin resistance, and virulence factors. Eur J Clin Microbiol Infect Dis 30: 499-508.

16. Monecke S, Coombs G, Shore AC, Coleman DC, Akpaka P, Borg M, Chow H, Ip M, Jatzwauk L, Jonas D, Kadlec K, Kearns A, Laurent F, O'Brien FG, Pearson J, Ruppelt A, Schwarz S, Scicluna E, Slickers P, Tan HL, Weber S, Ehricht R (2011) A field guide to pandemic, epidemic and sporadic clones of methicillin-resistant Staphylococcus aureus. PLoS ONE 6: e17936.

\section{Corresponding author}

\section{Patrick Basset}

Service of Hospital Preventive Medicine

Lausanne University Hospital

1011 Lausanne

Switzerland

Phone: +41213147621

Email: Patrick.Basset@chuv.ch

Conflict of interests: No conflict of interests is declared. 
Supplementary Items

Table 1-S: Characteristics of the 84 Algerian MRSA recovered in the Bologhine Ibn Ziri University hospital (Algiers, Algeria).

\begin{tabular}{|c|c|c|c|c|c|c|c|c|c|c|c|c|}
\hline Strain & $\begin{array}{l}\text { Year of } \\
\text { isolation }\end{array}$ & gender & Child/Adult & Service & Specimen & PVL & clfB & spa & mecA & SCCmec & International Clone & Antibiotic resistance profile ${ }^{a}$ \\
\hline 22579 & 2006 & $\mathrm{~F}$ & Adult & Out & Furuncle & pos & 563 & 26 & pos & $\mathrm{ND}^{\mathrm{b}}$ & European CA-MRSA (ST80) & $\mathrm{P}, \mathrm{OXA}, \mathrm{K}$ \\
\hline 22573 & 2006 & M & NA & NA & Wound & pos & 562 & 26 & pos & IV & European CA-MRSA (ST80) & $\mathrm{P}, \mathrm{OXA}, \mathrm{K}$ \\
\hline 22576 & 2006 & M & Adult & Medicine & Pus & pos & 560 & 26 & pos & IV & European CA-MRSA (ST80) & $\mathrm{P}, \mathrm{OX}, \mathrm{K}, \mathrm{TE}, \mathrm{FA}$ \\
\hline 22582 & 2006 & $\mathrm{~F}$ & Adult & Medicine & Furuncle & pos & 561 & 26 & pos & IV & European CA-MRSA (ST80) & $\mathrm{P}, \mathrm{OX}, \mathrm{K}, \mathrm{FA}, \mathrm{TE}$ \\
\hline 22581 & 2006 & M & Adult & Surgery & NA & pos & 46 & 26 & pos & IV & European CA-MRSA (ST80) & P, OX, TE, K, FA \\
\hline 22574 & 2006 & M & Adult & Medicine & Pus & neg & 28 & 30 & pos & IIImercury & Brazilian Clone (ST239) & $\mathrm{P}, \mathrm{OX}, \mathrm{K}, \mathrm{GM}, \mathrm{OFX}$ \\
\hline 22571 & 2006 & M & Adult & Emergency & Wound & neg & 366 & 252 & pos & IV & Other & $\mathrm{P}, \mathrm{OX}, \mathrm{K}, \mathrm{TE}$ \\
\hline 22583 & 2006 & M & Adult & Emergency & Wound & neg & 28 & 30 & pos & IIImercury & Brazilian Clone (ST239) & $\mathrm{P}, \mathrm{OX}, \mathrm{K}, \mathrm{TE}$ \\
\hline 22584 & 2006 & NA & NA & NA & NA & neg & 28 & 30 & pos & IIImercury & Brazilian Clone (ST239) & P,OXA,K,T,FA,E,SXT,OFX \\
\hline 22578 & 2006 & M & Adult & Emergency & Wound & neg & 28 & 30 & pos & IIImercury & Brazilian Clone (ST239) & $\mathrm{P}, \mathrm{OX}, \mathrm{K}, \mathrm{TE}, \mathrm{FA}, \mathrm{OFX}, \mathrm{SXT}$ \\
\hline 22575 & 2006 & M & Adult & Emergency & Wound & neg & 366 & 252 & pos & IV & Other & $\mathrm{P}, \mathrm{OX}, \mathrm{K}, \mathrm{TE}, \mathrm{FA}, \mathrm{OFX}, \mathrm{SXT}$ \\
\hline 22577 & 2006 & M & Adult & Emergency & Wound & neg & 28 & 30 & pos & IIImercury & Brazilian Clone (ST239) & $\mathrm{P}, \mathrm{OX}, \mathrm{K}, \mathrm{TE}, \mathrm{FA}, \mathrm{OFX}, \mathrm{SXT}$ \\
\hline 22572 & 2006 & NA & NA & NA & NA & neg & 366 & 252 & pos & IV & Other & $\mathrm{P}, \mathrm{OXA}, \mathrm{K}$ \\
\hline 22580 & 2006 & M & Adult & Out & Wound & pos & 7 & 26 & pos & IV & European CA-MRSA (ST80) & $\mathrm{P}, \mathrm{OXA}, \mathrm{K}$ \\
\hline 22593 & 2007 & $\mathrm{~F}$ & Adult & Out & Pus & pos & 415 & 26 & pos & IV & European CA-MRSA (ST80) & $\mathrm{P}, \mathrm{OXA}, \mathrm{TE}$ \\
\hline 22599 & 2007 & $\mathrm{~F}$ & Adult & Surgery & Blood & pos & 415 & 26 & pos & IV & European CA-MRSA (ST80) & $\mathrm{P}, \mathrm{OXA}, \mathrm{K}-\mathrm{TE}$ \\
\hline 22594 & 2007 & $\mathrm{~F}$ & Adult & NA & Pus & pos & 46 & 26 & pos & IV & European CA-MRSA (ST80) & $\mathrm{P}, \mathrm{OXA}, \mathrm{T}, \mathrm{K}, \mathrm{FA}$ \\
\hline 22595 & 2007 & $\mathrm{~F}$ & Adult & Out & Pus & pos & 415 & 26 & pos & IV & European CA-MRSA (ST80) & P,OXA,OFX-CM-SXT \\
\hline 22598 & 2007 & $\mathrm{~F}$ & Child & Pediatrics & Pus & pos & 559 & 26 & pos & IV & European CA-MRSA (ST80) & $\mathrm{P}, \mathrm{OXA}, \mathrm{K}$ \\
\hline 22596 & 2007 & $\mathrm{~F}$ & NA & NA & Pus & pos & 415 & 26 & pos & IV & European CA-MRSA (ST80) & $\mathrm{P}, \mathrm{OXA}, \mathrm{AF}-\mathrm{K}$ \\
\hline 22597 & 2007 & $\mathrm{~F}$ & NA & Out & Pus & pos & 415 & 26 & pos & IV & European CA-MRSA (ST80) & P,OXA,K-TE-FA \\
\hline 22600 & 2007 & M & Child & Pediatrics & Blood & pos & 415 & 26 & pos & IV & European CA-MRSA (ST80) & $\mathrm{P}, \mathrm{OXA}$ \\
\hline 22601 & 2007 & $\mathrm{~F}$ & Child & Pediatrics & Blood & pos & 415 & 26 & pos & IV & European CA-MRSA (ST80) & P,OXA,FA-SXT \\
\hline 22618 & 2008 & NA & NA & Surgery & NA & pos & 7 & 26 & pos & IV & European CA-MRSA (ST80) & P,OXA,FA,SXT \\
\hline 22613 & 2008 & $\mathrm{~F}$ & Child & Pediatrics & NA & pos & 559 & 26 & pos & IV & European CA-MRSA (ST80) & $\mathrm{P}, \mathrm{OXA}, \mathrm{K}, \mathrm{T}, \mathrm{FA}$ \\
\hline 22603 & 2008 & $\mathrm{~F}$ & Adult & Out & Nose & pos & 415 & 26 & pos & IV & European CA-MRSA (ST80) & $\mathrm{P}, \mathrm{OXA}, \mathrm{K}-\mathrm{FA}$ \\
\hline 22615 & 2008 & NA & NA & Surgery & NA & pos & 46 & 26 & pos & IV & European CA-MRSA (ST80) & $\mathrm{P}, \mathrm{OXA}, \mathrm{FA}$ \\
\hline 22604 & 2008 & $\mathrm{~F}$ & Adult & Out & Furuncle & pos & 415 & 26 & pos & IV & European CA-MRSA (ST80) & $\mathrm{P}, \mathrm{OXA}, \mathrm{K}$ \\
\hline 22617 & 2008 & M & Child & Out & Pus & pos & 46 & 26 & pos & IV & European CA-MRSA (ST80) & $\mathrm{P}, \mathrm{OXA}, \mathrm{K}$ \\
\hline 22612 & 2008 & NA & NA & NA & NA & pos & 559 & 26 & pos & IV & European CA-MRSA (ST80) & P,OXA,FA,SXT \\
\hline 22620 & 2008 & M & Adult & Surgery & Blood & pos & 415 & 26 & pos & IV & European CA-MRSA (ST80) & $\mathrm{P}, \mathrm{OXA}, \mathrm{SXT}$ \\
\hline 22616 & 2008 & NA & NA & NA & NA & pos & 559 & 26 & pos & IV & European CA-MRSA (ST80) & $\mathrm{P}, \mathrm{OXA}, \mathrm{K}-\mathrm{TE}$ \\
\hline
\end{tabular}


Table 1-S: continued

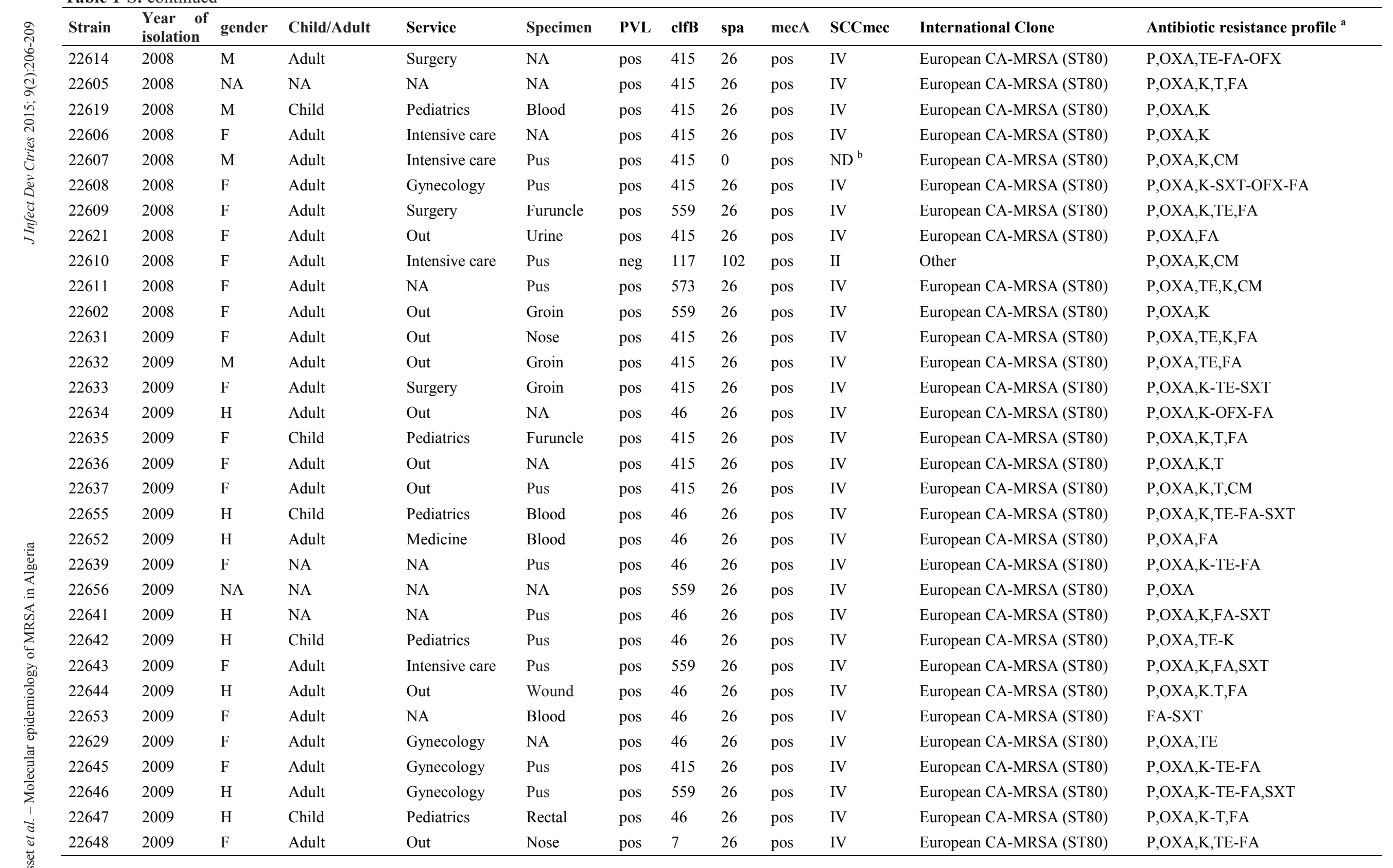


Table 1-S: continued

\begin{tabular}{|c|c|c|c|c|c|c|c|c|c|c|c|c|}
\hline Strain & $\begin{array}{l}\text { Year of } \\
\text { isolation }\end{array}$ & gender & Child/Adult & Service & Specimen & PVL & clfB & spa & mecA & SCCmec & International Clone & Antibiotic resistance profile ${ }^{a}$ \\
\hline 22649 & 2009 & $\mathrm{H}$ & Adult & Out & Nose & pos & 46 & 26 & pos & IV & European CA-MRSA (ST80) & $\mathrm{P}, \mathrm{OXA}, \mathrm{K}, \mathrm{T}, \mathrm{FA}$ \\
\hline 22654 & 2009 & $\mathrm{~F}$ & Adult & NA & Blood & pos & 46 & 26 & pos & IV & European CA-MRSA (ST80) & P,OXA,FA,CM \\
\hline 22630 & 2009 & M & Adult & Out & Groin & pos & 415 & 26 & pos & IV & European CA-MRSA (ST80) & P,OXA,K-TE-FA \\
\hline 22650 & 2009 & $\mathrm{~F}$ & Adult & Gynecology & Nose & pos & 46 & 26 & pos & IV & European CA-MRSA (ST80) & P,OXA,K,FA-CM-OFX-SXT \\
\hline 22651 & 2009 & $\mathrm{~F}$ & Adult & Gynecology & Pus & pos & 46 & 26 & pos & IV & European CA-MRSA (ST80) & $\mathrm{P}, \mathrm{OXA}, \mathrm{K}, \mathrm{T}, \mathrm{FA}-\mathrm{SXT}$ \\
\hline 22585 & 2010 & $\mathrm{~F}$ & Adult & Out & Nose & pos & 46 & 26 & pos & IV & European CA-MRSA (ST80) & $\mathrm{P}, \mathrm{OXA}, \mathrm{K}, \mathrm{T}, \mathrm{FA}$ \\
\hline 22590 & 2010 & $\mathrm{H}$ & Adult & NA & Pus & neg & 28 & 30 & pos & IIImercury & Brazilian Clone (ST239) & P,OXA,K-TE-OFX \\
\hline 22587 & 2010 & $\mathrm{H}$ & Adult & Out & Wound & pos & 415 & 26 & pos & IV & European CA-MRSA (ST80) & P,OXA,TE \\
\hline 22588 & 2010 & $\mathrm{~F}$ & Child & Out & Wound & pos & 415 & 26 & pos & IV & European CA-MRSA (ST80) & $\mathrm{P}, \mathrm{OXA}, \mathrm{TE}$ \\
\hline 22592 & 2010 & $\mathrm{H}$ & Adult & Intensive care & Blood & neg & 28 & 30 & pos & IIImercury & Brazilian Clone (ST239) & P,OXA,K-OFX \\
\hline 22591 & 2010 & $\mathrm{~F}$ & Adult & Gynecology & Pus & pos & 46 & 26 & pos & IV & European CA-MRSA (ST80) & P,OXA,FA-SXT \\
\hline 22628 & 2010 & $\mathrm{H}$ & Adult & Intensive care & Pus & pos & 7 & 26 & pos & IV & European CA-MRSA (ST80) & $\mathrm{P}, \mathrm{OXA}, \mathrm{K}, \mathrm{FA}$ \\
\hline 22589 & 2010 & $\mathrm{~F}$ & Adult & NA & Pus & pos & 46 & 26 & pos & IV & European CA-MRSA (ST80) & $\mathrm{P}, \mathrm{OXA}, \mathrm{K}, \mathrm{FA}, \mathrm{CM}$ \\
\hline 23878 & 2011 & M & Adult & Medicine & Blood & pos & 7 & 26 & pos & IV & European CA-MRSA (ST80) & $\mathrm{P}, \mathrm{OXA}, \mathrm{K}, \mathrm{T}, \mathrm{FA}$ \\
\hline 23872 & 2011 & M & Adult & Medicine & Blood & pos & 7 & 26 & pos & IV & European CA-MRSA (ST80) & $\mathrm{P}, \mathrm{OXA}, \mathrm{K}, \mathrm{T}, \mathrm{FA}$ \\
\hline 23879 & 2011 & M & Adult & Intensive care & Blood & pos & 7 & 26 & pos & IV & European CA-MRSA (ST80) & $\mathrm{P}, \mathrm{OXA}, \mathrm{K}, \mathrm{T}, \mathrm{FA}$ \\
\hline 23873 & 2011 & $\mathrm{~F}$ & Adult & NA & Pus & pos & 415 & 26 & pos & IV & European CA-MRSA (ST80) & $\mathrm{P}, \mathrm{OXA}, \mathrm{K}, \mathrm{T}, \mathrm{FA}$ \\
\hline 23874 & 2011 & M & Adult & Medicine & Pus & pos & 7 & 26 & pos & IV & European CA-MRSA (ST80) & $\mathrm{P}, \mathrm{OXA}, \mathrm{K}, \mathrm{T}, \mathrm{FA}$ \\
\hline 23876 & 2011 & $\mathrm{~F}$ & Adult & Gynecology & Pus & pos & 46 & 26 & pos & IV & European CA-MRSA (ST80) & $\mathrm{P}, \mathrm{OXA}, \mathrm{K}, \mathrm{T}, \mathrm{FA}$ \\
\hline 23877 & 2011 & $\mathrm{~F}$ & NA & Out & Groin & neg & 2 & 0 & pos & IV & Other & $\mathrm{P}, \mathrm{OXA}, \mathrm{K}, \mathrm{T}, \mathrm{FA}, \mathrm{SXT}$ \\
\hline
\end{tabular}

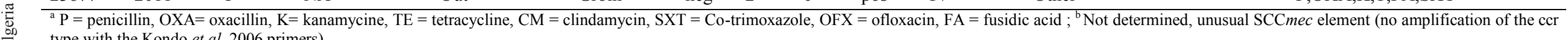
type with the Kondo et al. 2006 primers) 
Table 2-S: Presence/absence of resistance- (A) and virulence- (B) associated genes as detected with the Alere StaphyType DNA microarray (Monecke et al. 2011, PLoS One 6(4):

\begin{tabular}{|c|c|c|c|c|c|c|c|}
\hline (A) & $\begin{array}{l}\text { European CA- } \\
\text { MRSA (22599) } \\
\end{array}$ & $\begin{array}{l}\text { European CA- } \\
\text { MRSA (22654) } \\
\end{array}$ & $\begin{array}{c}\text { Brazilian Clone } \\
(22590)\end{array}$ & (B) & $\begin{array}{l}\text { European CA- } \\
\text { MRSA (22599) } \\
\end{array}$ & $\begin{array}{l}\text { European CA- } \\
\text { MRSA (22654) } \\
\end{array}$ & $\begin{array}{c}\text { Brazilian Clone } \\
(22590) \\
\end{array}$ \\
\hline blaZ & neg & pos & pos & PVL genes & pos & pos & neg \\
\hline $\operatorname{erm}(A)$ & neg & neg & pos & $A C M E$ & neg & neg & neg \\
\hline $\operatorname{erm}(B)$ & neg & neg & neg & sak & pos & pos & pos \\
\hline $\operatorname{erm}(C)$ & neg & neg & neg & $\operatorname{ch} p$ & neg & neg & pos \\
\hline $\ln u(A)$ & neg & neg & neg & scn & pos & pos & neg \\
\hline$m s r(A)$ & neg & neg & neg & sea & neg & neg & neg \\
\hline$m p h(C)$ & neg & neg & neg & seb & neg & neg & neg \\
\hline $\operatorname{vg} a(A)$ & neg & neg & neg & sek \& seq & neg & neg & pos \\
\hline$a a c A-a p h D$ & neg & pos & pos & $\sec \&$ sel & neg & neg & neg \\
\hline$a a d D$ & neg & neg & neg & tst 1 & neg & neg & neg \\
\hline aphA3 \&sat & pos & pos & pos & sed & neg & neg & neg \\
\hline$d f r A$ & neg & neg & neg & sej \& ser & neg & neg & neg \\
\hline farl & neg & neg & neg & egc-genes & neg & neg & neg \\
\hline Q6GD50 & neg & neg & neg & seh & neg & neg & neg \\
\hline тирА & neg & neg & neg & etA & neg & neg & neg \\
\hline $\operatorname{tet}(K)$ & neg & neg & neg & $e t D$ & pos & pos & neg \\
\hline $\operatorname{tet}(M)$ & neg & neg & pos & $e \operatorname{din} A$ & neg & neg & neg \\
\hline cat & neg & neg & neg & $e \operatorname{din} B$ & pos & pos & neg \\
\hline$c f r$ & neg & neg & neg & splA \& splB & pos & pos & neg \\
\hline fexA & neg & neg & neg & hld & neg & neg & pos \\
\hline$q a c A$ & neg & neg & neg & aur & neg & neg & pos \\
\hline$q a c C$ & neg & neg & neg & & & & \\
\hline merc $A \&$ mer $B$ & neg & neg & neg & & & & \\
\hline fos $B$ & neg & neg & pos & & & & \\
\hline
\end{tabular}

